\title{
FLARE-TYPE EVENTS AND GENERATION OF PLASMA JETS IN CURRENT SHEETS EVOLVING IN LABORATORY CONDITIONS
}

\author{
A.G. Frank ${ }^{1}$
}

\begin{abstract}
Laboratory experiments demonstrate that current sheets evolving in magnetized plasmas can give rise to both flare-type events and generation of energetic plasma jets.
\end{abstract}

\section{Introduction}

Coronal mass ejections (CMEs) and solar flares are the most dramatic manifestations of the solar activity. Both types of events occur in the active areas of the Sun, in strong magnetic fields with complicate structure. The energy liberated during CMEs and flares is the energy of non-potential magnetic fields, i.e. the energy of electric currents in the solar corona. Flares and CMEs have been investigated for many decades in numerous astrophysical observations. At the same time, the laboratory experiments performed in well controlled and reproducible conditions, by using modern methods of plasma diagnostics, may contribute to better understanding of the physical nature and interplay of CMEs and flares.

According to present notion, magnetic reconnection in the current sheets (CSs) is a basis to many non-stationary phenomena in astrophysics as well as in laboratory devices. We present a brief review of the experimental research of the CS dynamics; the results demonstrate that the flare-type events and generation of plasma jets may be realized in the laboratory produced CSs.

\section{Experimental device CS-3D and current sheet formation}

The experiments are performed with the device CS-3D, Figure 1, at the Institute of General Physics, Moscow (Frank 1999; Bogdanov et al. 2000). Three independent power supply systems operate sequentially producing: a quasi-steady $3 \mathrm{D}$ magnetic

1 A.M. Prokhorov Institute of General Physics of the Russian Academy of Sciences, 38 Vavilov Str., Moscow 119991, Russia; e-mail: annfrank@fpl.gpi.ru 
configuration with a singular line of the $X$ type; initial plasma in this magnetic field; and, finally, electric current along the $X$ line, which gives rise to a current sheet. The initial (current-free) magnetic field is produced by the currents in external coils and straight conductors. This field is characterized by the transverse (in the (x, y) plane) gradient $h=(0.5-2) \mathrm{kG} / \mathrm{cm}$ and the uniform longitudinal (guide) field, $B_{z}^{0}=(0-4.5) \mathrm{kG}$ :

$$
\mathbf{B}=\left\{B_{x}^{0} ; B_{y}^{0} ; B_{z}^{0}\right\}=\left\{h y ; h x ; B_{z}^{0}\right\}
$$

The structure of the transverse components $\left(B_{x}^{0}, B_{y}^{0}\right)$ is shown in Figure $1 \mathrm{~b}$, and the $X$ line of the field (2.1) is aligned with the axis of the quartz vacuum chamber $18 \mathrm{~cm}$ in diameter and $100 \mathrm{~cm}$ long, Figure 1. The initial plasma with the

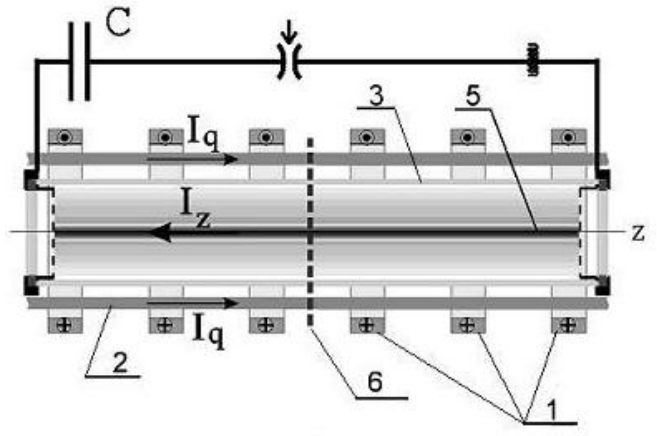

a

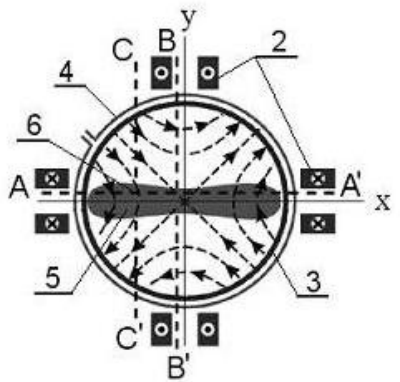

b

Fig. 1. Device CS-3D: (a) side view, (b) cross section; (1) coils to produce the guide field $B_{z},(2)$ conductors to produce $2 \mathrm{D}$ transverse magnetic field with a null line at the $z$-axis and the field lines in the $(x, y)$ plane, (3) vacuum chamber, (4) $\Theta$-discharge coils, (5) current sheet, (6) lines AA', BB', CC' show trajectories of mobile magnetic probes.

electron density $N_{e} \approx\left(2 \cdot 10^{14}-5 \cdot 10^{15}\right) \mathrm{cm}^{-3}$ is produced by $\Theta$-discharge. In the prepared plasma, the current $J_{z}$ is excited by applying a pulsed voltage between two electrodes located at a distance $\Delta \mathrm{z}=60 \mathrm{~cm}$ from each other, Figure 1a. The current amplitude is up to $\approx 70 \mathrm{kA}$, its half period is $6 \mu \mathrm{s}$. The CS forms usually within $1-1.5 \mu$ s after exciting the current $J_{z}$. The CS characteristic dimensions are: length $\Delta \mathrm{z}$, width $2 \delta \mathrm{x} \approx(8-12) \mathrm{cm}$, and thickness $2 \delta \mathrm{y} \approx(1.5-3.5) \mathrm{cm}$. Plasma current concentration in the CS modifies the initial configuration (2.1): the tangential component $B_{x}$ increases significantly, while the normal component $B_{y}$ decreases as compared to their initial values, $B_{x}^{0}, B_{y}^{0}$ (see Fig. 1b and Fig. 2), and the guide field $B_{z}$ in the CS exceeds $B_{z}^{0}$ (Frank \& Bogdanov 2001; Frank et al. 2008, 2009). The CS formation results also in effective plasma compression into the sheet, so that the electron density in the CS mid-plane increases up to $(1-3) \cdot 10^{16} \mathrm{~cm}^{-3}$, i.e., it is 5-15 times the initial density (Bogdanov et al. 2002; Frank et al. 2005). 


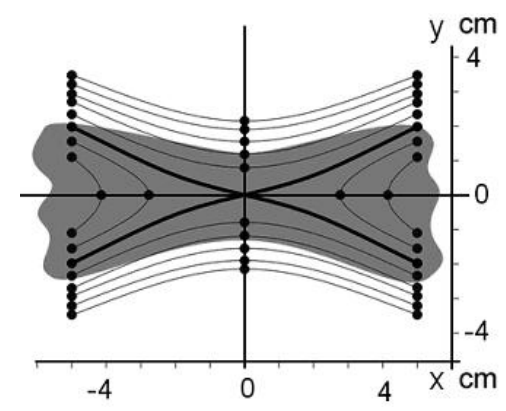

Fig. 2. Structure of the transverse magnetic field of the CS; separatrices are shown by the bold lines (Frank et al. 2008).
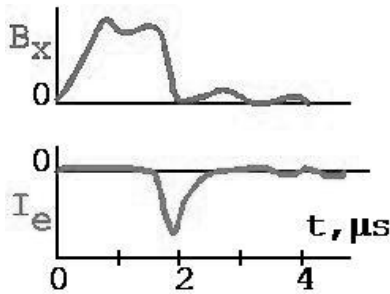

Fig. 3. Time dependences of the tangential magnetic field component $B_{x}$ and the hard X-ray (energy $\geq 10 \mathrm{keV}$ ) intensity. At $t=$ $1.8 \mu$ s an abrupt decay of $B_{x}$ indicates the onset of the impulsive phase of magnetic reconnection accompanied by acceleration of electrons.

\section{Flare-type events in current sheets}

After the CS forms, it usually exists during an extended period in a metastable stage, without essential change in its structure and parameters; both the current and plasma are concentrated in the sheet; magnetic reconnection occurs at a relatively slow rate; the tearing-mode instability has no pronounced effect on the CS evolution (Frank \& Bogdanov 2001). The electron and ion temperatures, as well as the effective ion charge $Z_{i}$, are maximum in the CS mid-plane. Their values are gradually increasing with time (Voronov et al. 2008). Throughout the metastable stage, we have $T_{e} \approx(5-15) \mathrm{eV}, T_{i} \approx(20-100) \mathrm{eV}$, and $T_{i}>T_{e}$. The hot and dense plasma inside the $\mathrm{CS}$ is in a transverse equilibrium with the magnetic field outside:

$$
N_{e}\left(T_{e}+T_{i} / Z_{i}\right)+\left(\delta B_{z}\right)^{2} / 8 \pi \cong B_{x}^{2} / 8 \pi ; \quad \beta \leq 1
$$

Under certain conditions, the metastable stage may be suddenly interrupted by an impulsive phase of magnetic reconnection, which shows itself in rapid changes of the magnetic field topology, conversion of excessive magnetic energy into the energy of plasma and accelerated particles. Finally, the impulsive phase brings about a disruption of the CS (Frank 2010). In the course of the impulsive phase the electrons are accelerated by inductive electric fields owing to fast changes of the magnetic field, Figure 3. A long-standing problem is to find out the physical mechanisms responsible for interrupting the metastable stage and the onset of the impulsive phase. Evolution of the electron and ion temperatures shows that the thermal energy is rapidly built up in a small magnetic island inside the CS, just before the impulsive phase, Figure 4 (Kirii et al. 1992). As a result, the CS transverse equilibrium breaks down, and the impulsive phase comes to play. The role of thermal processes in the origin of solar flares was discussed by Coppi (1975) and Syrovatskii (1976). 


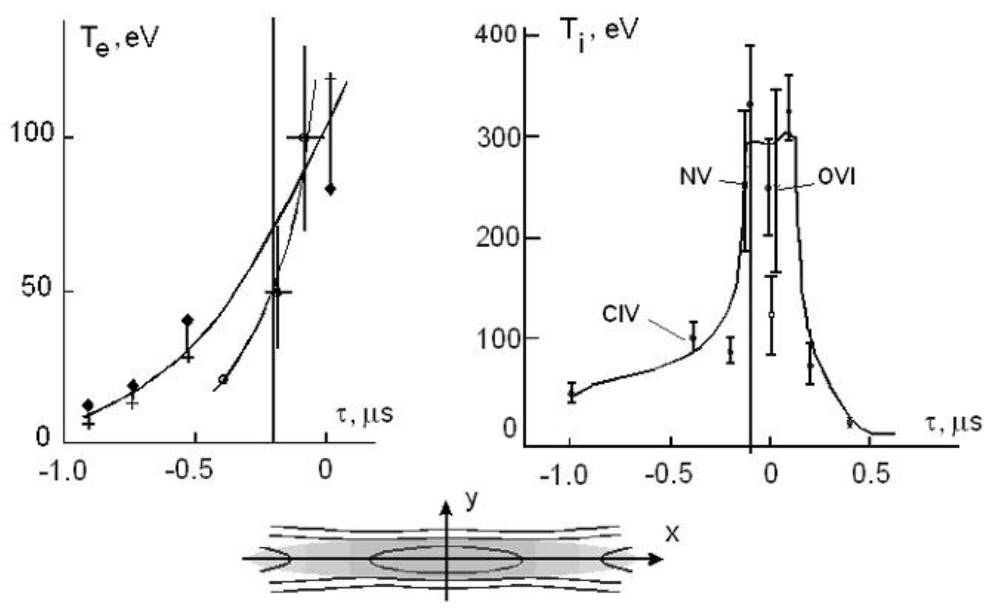

Fig. 4. Time evolution of the electron and ion temperatures; here $\tau=0$ corresponds to the onset of the impulsive phase. The magnetic structure of the current sheet with a magnetic island inside is shown at the bottom (Kirii et al. 1992).

Hence, the impulsive phase of magnetic reconnection in the laboratory produced CSs displays principal features of the flare-type phenomena, including fast magnetic energy release, intense plasma heating, mass ejections, and acceleration of charged particles. In a context of solar flares, the metastable CS should be treated as a pre-flare situation, while the flare itself might be associated with a fast CS disruption (Syrovatskii 1981).

\section{Generation of plasma jets in current sheets}

Measurements of magnetic fields and plasma currents in the CSs make it possible to calculate the forces, which can accelerate plasma and generate the outflow plasma jets, Figure 5 (Frank \& Satunin 2011). Plasma motion is governed by both the pressure gradient and the Ampere force:

$$
\left(M_{i} \cdot N_{i}\right) \cdot d \mathbf{v} / d t=-\nabla p+1 / c \cdot[\mathbf{j} \times \mathbf{B}] .
$$

The Ampere forces acting along the normal to the CS surface ( $y$-axis) result in the current and plasma compression. The pressure gradient impedes compression and the CS formation brings about the establishment of transverse equilibrium. The pressure gradient is negligible along the CS surface ( $x$-axis), whereas the Ampere forces are of crucial importance for the Hall current excitation (Frank et al. 2008) and the generation of plasma jets (Kyrie et al. 2010). The Ampere force $[\mathbf{j} \times \mathbf{B}]_{x}$ along the CS width is determined by current $j_{z}$ and normal component $B_{y}$, which is usually nonzero in the metastable CS (Fig. 2). The Ampere forces $F_{x}$ on each side of the $X$ line are oppositely directed, as shown in Figure 5. 


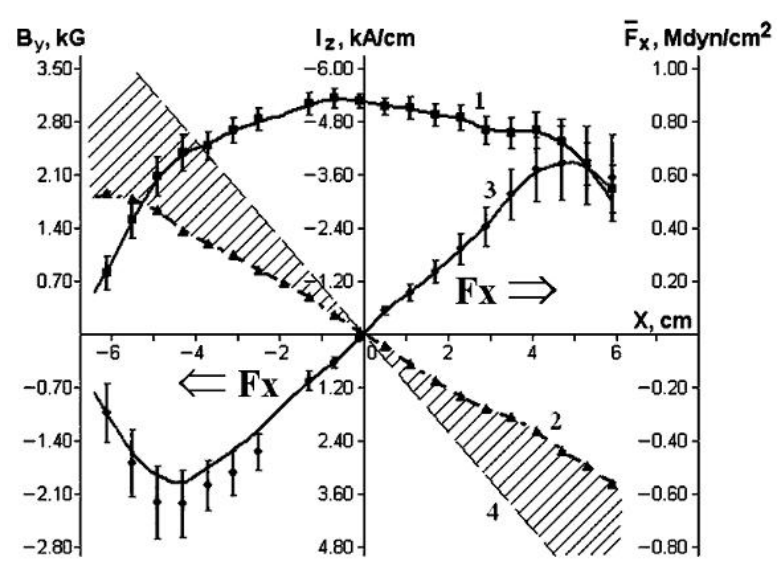

Fig. 5. Distributions of $I_{z}(x)$ inside the area $|y| \leq \Delta y=1.2 \mathrm{~cm}$ (1); the normal magnetic field component $B_{y}^{J}(x)$ produced by plasma currents and measured by a magnetic probe (2); the Ampere force $F_{x}(x)$ averaged over the area $2 \Delta y(3)$; the function $\left\{-B_{y}^{0}(x)\right\}=-h$. $x$ (4). The height of the shaded region between (4) and (2) is the normal component $\left\{-B_{y}(x)\right\}=\left(B_{y}^{0}+B_{y}^{J}\right)$, which produces the Ampere force $F_{x}(x)$ (Frank \& Satunin 2011).

Outflowing energetic plasma jets produced in the CSs are detected using spectroscopic methods (Kyrie et al. 2010). The averaged energy of plasma jets increases with time, reaching $100 \mathrm{eV}$ in the Ar plasma, whereas the ion temperature does not exceed $45 \mathrm{eV}$. A correlation was found between the measured jet energies and the calculated work of the Ampere forces over a half width of the CSs produced in the Ar plasma.

In the CSs, which are formed in He plasma, the ion temperature is (50-90) eV, whereas plasma jets gain much higher energies: from 400 to $1300 \mathrm{eV}$, depending on the initial conditions, see Figure 6 as an example (Kyrie et al. 2012). It is suggested that plasma acceleration is more effective in the regions with lower plasma density, which are located at some distance from the CS midplane (Frank et al. 2011).

Generation of plasma jets is also confirmed by observation of reverse currents, which flow at the CS side edges in a direction opposite to the main electric current through the system (Frank \& Satunin 2011). The values of reverse currents increase with time, and the regions where they are localized expand gradually from the edges toward the CS center, Figure 7 (Frank et al. 2011). Reverse electric fields and currents are caused by high-speed plasma jets moving along the $x$-axis toward the CS edges, where $B_{y}$ component is strong enough. We emphasize that current sheets containing reverse currents were predicted by Syrovatskii (1971).

This work is supported by the Russian Foundation for Basic Research (project No. 12-02-00553a) and the Program (OFN-15) "Plasma Processes in Space and Laboratory" of the Division of Physical Sciences of the Russian Academy of Sciences. 


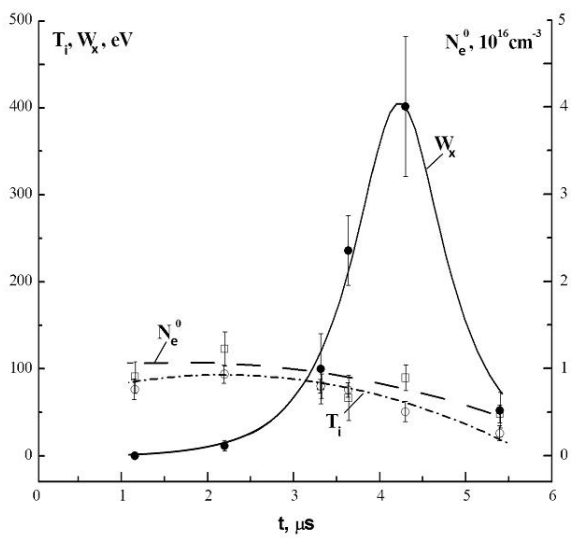

Fig. 6. Time dependences of the electron density $N_{e}$ and the ion temperature $T_{i}$ in the central part of the CS formed in the He plasma; $W_{x}$ - averaged energy of plasma jets (Frank et al. 2011).

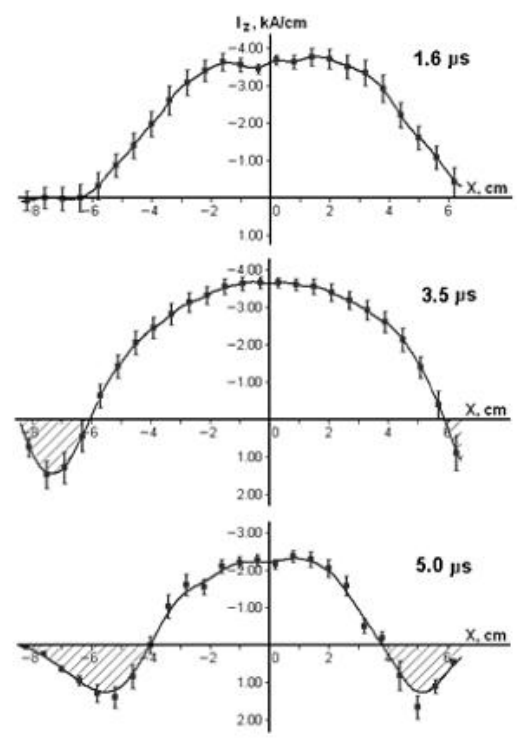

Fig. 7. Distributions of $I_{z}(x)$ inside the area $|y| \leq \Delta y=0.8 \mathrm{~cm}$ at times $t=1.6$, $3.5,5.0 \mu \mathrm{s}$. The regions with reverse currents are shaded (Frank \& Satunin 2011).

\section{References}

Bogdanov, S.Yu., Kyrie, N.P., Markov, V.S., \& Frank, A.G., 2000, JETP Lett., 71, 53 Bogdanov, S.Yu., Markov, V.S., Frank, et al., 2002, Plasma Phys. Rep., 28, 549 Frank, A.G, 1999, Plasma Phys. \& Control. Fusion, 41, Suppl. 3A, A687 Frank, A.G, 2010, Physics-Uspekhi, 180, 941

Coppi, B., 1975, ApJ, 195

Frank, A.G., \& Bogdanov, S.Yu., 2001, Earth, Planets \& Space (EPS), 53, 531

Frank, A.G., Bogdanov, S.Yu., Markov, et al., 2005, Phys. Plasmas, 12, 052316

Frank, A.G., Bugrov, S.G., \& Markov, V.S., 2008, Phys. Plasmas, 15, 092102

Frank, A.G., Bugrov, S.G., \& Markov, V.S., 2009, Phys. Lett. A, 373, 1460

Frank, A.G., \& Satunin, S.N., 2011, Plasma Phys. Rep., 37, 829

Frank, A.G., Kyrie, N.P., \& Satunin, S.N., 2011, Phys. Plasmas, 18, 111209

Kirii, N.P., Markov, V.S., \& Frank, A.G., 1992, JETP Lett., 56, 82

Kyrie, N.P., Markov, V.S., \& Frank, A.G., 2010, Plasma Phys. Rep., 36, 357

Kyrie, N.P., Markov, V.S., \& Frank, A.G., 2012, JETP Lett., 95, 17

Syrovatskii, S.I., 1971, Sov. Phys. JETP, 33, 933

Syrovatskii, S.I., 1976, Sov. Astron. Lett., 2, 13

Syrovatskii, S.I., 1981, ARA\&A, 19, 163

Voronov, G.S., Kyrie, N.P., Markov, et al., 2008, Plasma Phys. Rep., 34, 999 